\section{Massive Pulmonary Embolism Associated with Hemorrhagic Colitis: what is the Link? \\ CASE REPORT}

\section{Abstract}

Tittle: Deep vein thrombosis in the lower limbs and pulmonary embolism are the most common thromboembolic phenomena in patients with inflammatory bowel disease. The inflammatory and thrombotic processes are connected, creating a vicious circle. Inflammation is at least implicated in maintaining the inflammatory process in ulcerative colitis and Crohn's disease. Many factors have been investigated as determinants of the pro-thrombotic tendency such as acquired risk factors or genetic and immune abnormalities, but a unique cause has not been found.

Background: We felt that this case was of interest due to the rarity of the condition and the therapeutic challenge given the high risk of bleeding and the management of anti-inflammatory and immunological treatment.

Case presentation: We describe the rare case of a patient with ulcerative colitis who suffered a massive pulmonary embolism that caused shock. Despite the therapeutic challenge and the high risk of hemorrhagic complications given the underlying disease, thrombolytic treatment was administered with a good result.

Conclusion: Autoimmune disorders in general should be regarded not only as inflammatory disorders, but also as hyper-coagulable disorders. This clinical observation can put the item on the necessity of establishing recommendations for prevention of embolic events in patients with hemorrhagic colitis.

\author{
Sahar Mouram", \\ Hicham Sabor ${ }^{2}$, \\ Imane Mouram 3 \\ 1 Cardiology Department, Military \\ Hospital, Faculty of Medicine and \\ Pharmacy, Rabat, Morocco. \\ 2 Cardiology Department, Military \\ Hospital, Faculty of Medicine and \\ Pharmacy, Rabat, Morocco. \\ 3 Biology Department, Faculty of \\ Pharmacy, Seville, Spain.
}

\section{Contact information:}

\section{Sahar Mouram.}

Cardiology Department.

Address: Military Hospital, Faculty of Medicine and Pharmacy, Rabat, Morocco. Tel: 00212(0) 661630785.

Đmouram.sahar@gmail.com

\title{
Keywords
}

Hemorrhagic Colitis; Pulmonary Embolism; Thrombolysis. 


\section{Introduction}

The inflammatory and thrombotic processes are connected in patients with inflammatory bowel disease. We felt that this case was of interest due to the rarity of the condition and the therapeutic challenge given the high risk of bleeding and the management of anti-inflammatory and immunological treatment. This clinical observation can put the item on the necessity of establishing recommendations for prevention of embolic events in patients with hemorrhagic colitis.

\section{Case report}

A women aged 56 years came to our department because of dyspnea on effort that had progressively worsened over three days. She had a history of ulcerative colitis, diagnosed by colonoscopy and biopsy of the end section of the large bowel seven years previously. The patient had been under treatment with prednisone and mesalazine in daily dosages of $20 \mathrm{mg}$ and $750 \mathrm{mg}$, respectively, with periodic remission of the disease. The last acute thrust occurred one month earlier. The clinical examination on admission showed a rhythmic, rapid, and narrow arterial pulse; blood pressure was $70 / 50 \mathrm{mmHg}$ and heart rate was 140/min. The patient exhibited tachypnea (30 breaths/min) and diaphoresis. Her saturation was $89 \%$ improved with high concentration mask. Peripheral pulses were equally palpable at all sites. The lower limbs showed no edema and the skin had a normal temperature, without redness or cyanosis. Auscultation of the lungs revealed a small reduction in the respiratory murmur bilaterally. On cardiac auscultation the heart sounds were distinct, with audible third and fourth sounds and an accentuated pulmonary second sound. In reclined position there was an increase in the pulse amplitude in the internal jugular. The internal organs were not palpable. The ECG showed sinus rhythm at a rate of 140/min, an appearance S1Q3. Chest X-ray in the postero-anterior projection showed normal cardiac dimensions and protrusion of the left pulmonary artery conus. Blood gases showed hypoxemia and hypocapnia. On the transthoracic echocardiogram (Figure 1) the right ventricle was enlarged and had a significant degree of hypokinesia of its free wall, with a concomitant change in its triangular structure and a fresh thrombus attached to the apex of the right ventricle measuring $21 \mathrm{~mm} / 17 \mathrm{~mm}$. cutting para sternal minor axis showed the presence of a thrombus mobile expenses at the right bronchus pulmonary artery (Figure 2). Systolic pulmonary artery pressure, estimated from the tricuspid valve regurgitation, was $65 \mathrm{mmHg}$. The patient's clinical history and other examinations were strongly suggestive of pulmonary embolism, so a computed tomographic examination with intravenous administration of contrast medium was scheduled. This revealed the presence of filling defects in the main branches of the pulmonary arteries bilaterally, in segmental and lobular branches, but no pleuritic fluid accumulation or swollen lymph glands (Figure 3). An ultrasound examination of the lower limb vasculature didn't find deep vein thrombosis. The laboratory tests on admission showed: $\mathrm{Ht}$ : $42 \%$, $\mathrm{Hb}: 12.3 \mathrm{~g} / \mathrm{dl}, \mathrm{WBC}: 8500 / \mathrm{mm}^{3}$, PLT: 263000/ mm³, ESR: 20 mm/h, BNP: 315 pg/ml, CPK: $33 \mathrm{U} / \mathrm{L}$, troponin-l: $0.03 \mathrm{ng} / \mathrm{ml}$, fibrinogen: $0.80 \mathrm{~g} / \mathrm{l}, \mathrm{D}$-dimer: $5.0 \mathrm{mg} / \mathrm{l}$, CRP: $3.5 \mathrm{mg} / \mathrm{dl}$. Other values were normal. Given the diagnosis and the patient's hemodynamic instability, thrombolysis with alteplase was initial, as well as anticoagulation therapy with intravenous standard heparin (aiming at a partial thromboplastin time of 2-2.5 times the control value) and vasoactive drugs, while at the same time prednisone was continued at the same dosage the patient had been taking. The computed tomography image after thrombolysis are shown in Figure 4. Computed tomography of the chest showed a clear reduction in filling defects in the main branches of the pulmonary artery especially on the left, resulting in a reduction in the haemodynamic load 
Figure 1: Transthoracic echocardiogram para sternal cut 4 cavities centered on the right cavities; this cut shows the presence of an enlarged right ventricle with a fresh thrombus attached at the apex.

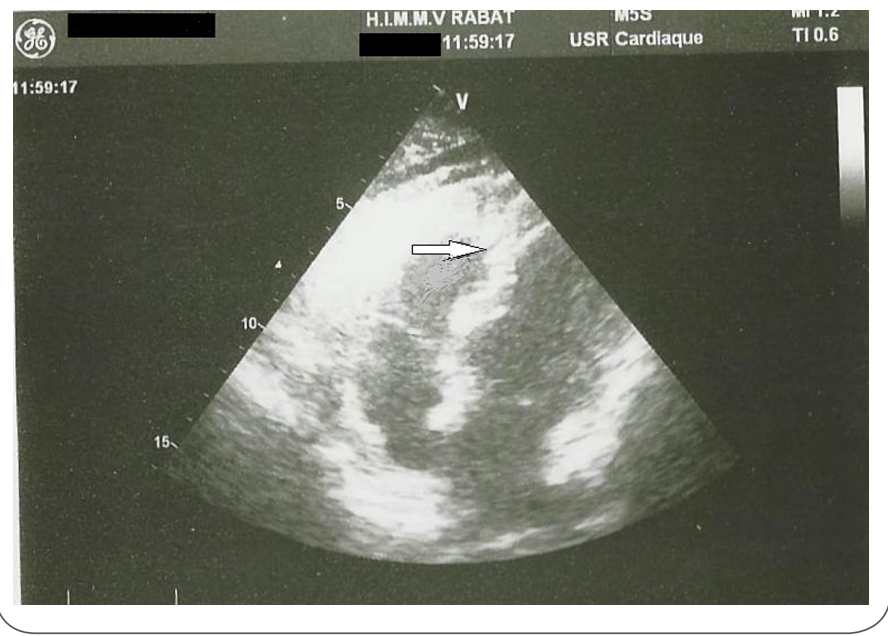

Figure 3: Computed tomographic examination with intra venous administration of contrast medium before thrombolysis; This revealed the presence of filling defects in the main branches of the pulmonary arteries bilaterally, in segmental and lobular branches.

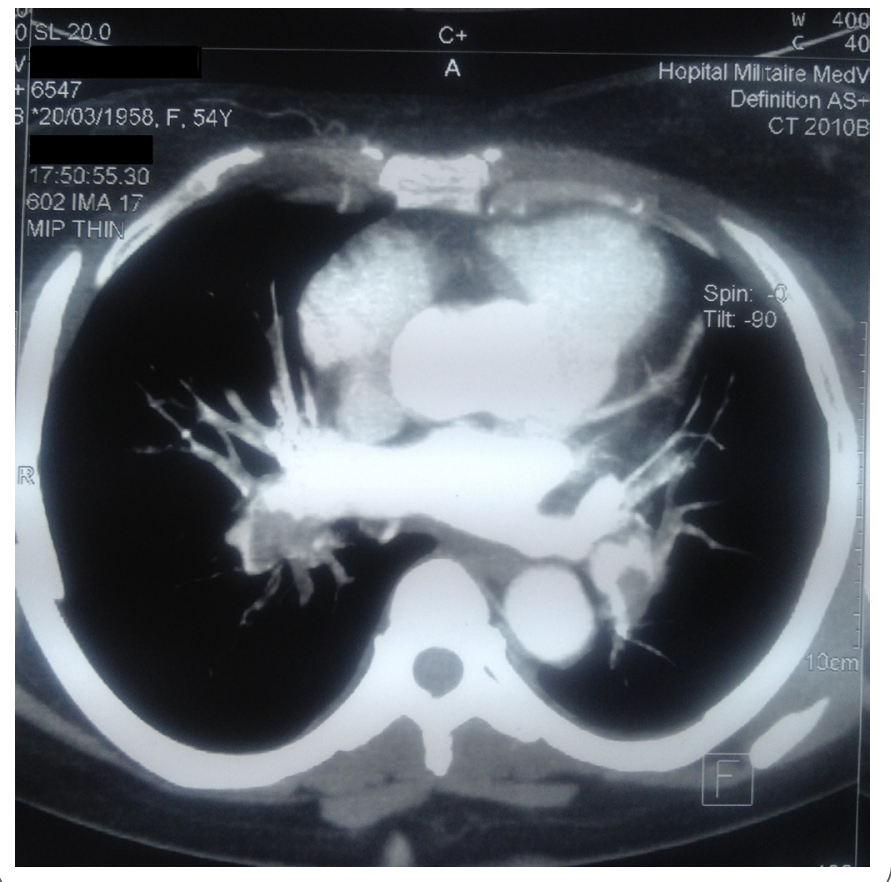

Figure 2: Transthoracic echocardiogram cutting para sternal minor axis; this cut shows the presence of a thrombus mobile expenses at the right bronchus pulmonary artery.

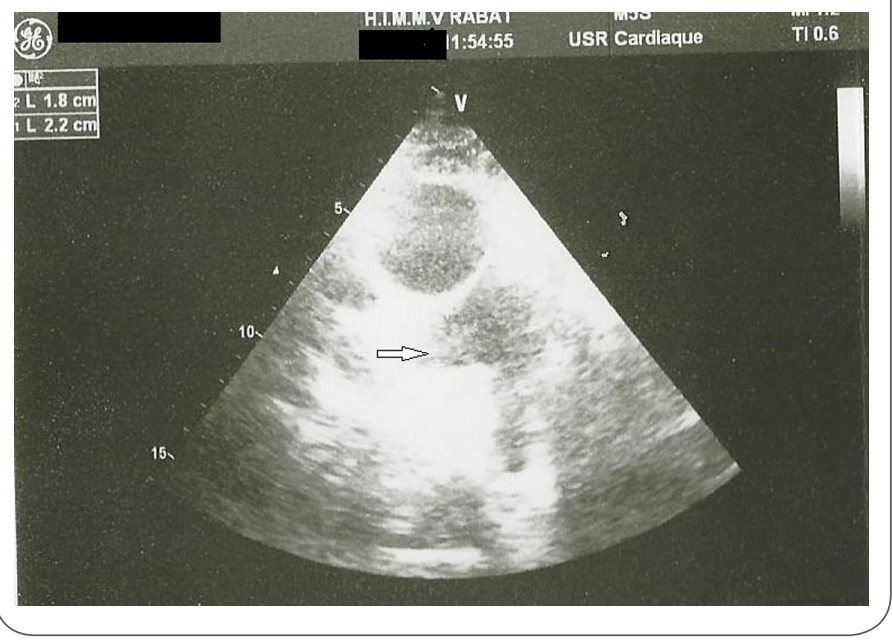

Figure 4: Computed tomographic examination with intra venous administration of contrast medium after thrombolysis; This showed a reduction in filling defects in the main branches of the pulmonary artery especially on the left, resulting in a reduction in the hemodynamic load of the right ventricle and correspondingly in heart rate.

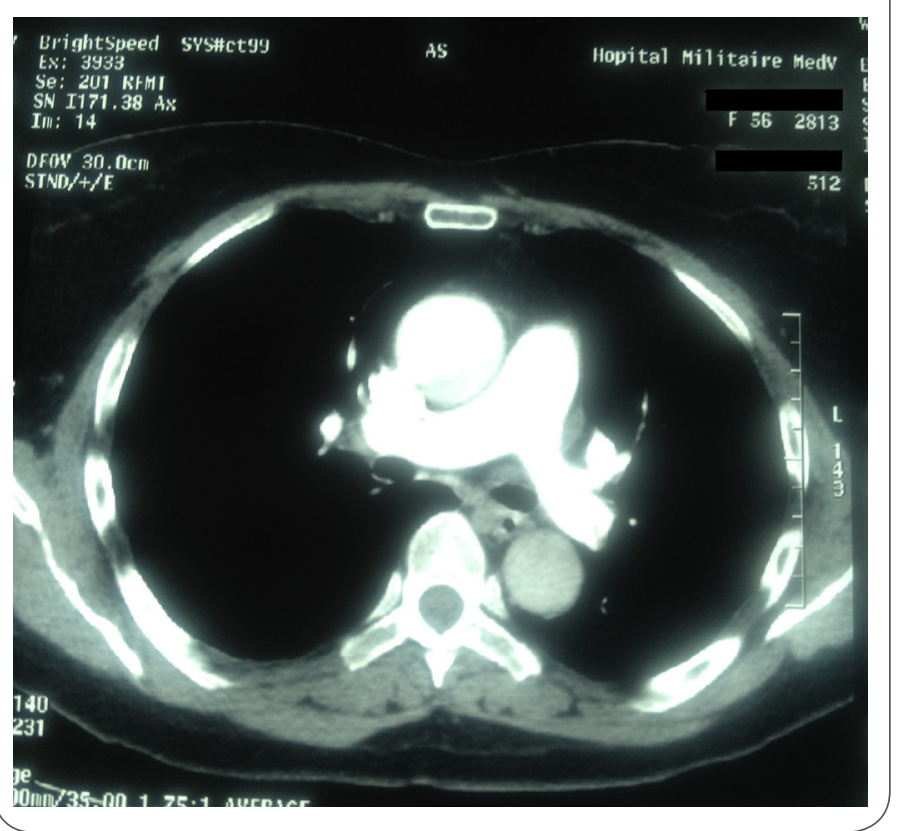


of the right ventricle and correspondingly in heart rate. The patient responded to treatment without presenting any complications and after his improvement was discharged in a good general condition, taking AVK anticoagulants according to the guidelines for the treatment and prevention of pulmonary embolism. The others causes of pulmonary embolism were sought.

\section{Discussion}

Venous thromboembolism is a major health problem [1] for which pulmonary embolism is a potentially lethal complication. Pulmonary embolism is a common cardiovascular and cardiopulmonary illness with a yearly incidence in the USA of more than one case per 1000 people and a mortality rate of more than $15 \%$ in the first 3 months after diagnosis [2] which is similar to that for acute myocardial infarction [3]. Autoimmune disorders are associated with a high risk of pulmonary embolism in the first year after hospital admission. Previous studies of the association between venous thromboembolism and autoimmune diseases have often been small and done mostly in individual autoimmune diseases.

Inflammatory bowel disease (IBD), whose major forms are ulcerative colitis (UC) and Crohn's disease (CD), is a chronic inflammatory condition characterized by local and systemic inflammation predominantly affecting the gastrointestinal tract but that may be associated to numerous extra-intestinal manifestations including thrombosis which is not a rare complication with high mortality rate. A serious and potentially fatal complication is pulmonary embolism resulting from thrombosis of the leg or pelvic veins. Inflammatory bowel disease (IBD) patients are at increased risk for venous thromboembolism (VTE) compared to the general population.

The literature reports few cases of thrombolysis in patients with IBD who had deep vein thrombosis and/or massive pulmonary embolism [4, 5]. In one case a transfusion was required because of massi- ve hemorrhage [5] Active IBD (versus inactive) may be a risk factor for thromboembolism, since active disease is associated with increased coagulability [6, 7] However, one third of thromboembolic complications occur in patients with non-active disease.

Many factors have been investigated as determinants of the pro-thrombotic tendency such as acquired risk factors or genetic and immune abnormalities, but a unique cause has not been found. The acquired factors include fluid depletion, prolonged immobilization, surgery, the use of central venous catheters, steroid therapy, oral contraceptives or hormone replacement therapy and cigarette smoking.

In spite of the big effort in terms of numerous genetic studies performed, no significant association has been demonstrated to date between genetic factors cause of hyper-coagulability and IBD. Thus, genetics of thrombophilia do not explain the increased TE risk in CD and UC patients, suggesting a more relevant role of acquired factors.

Many efforts have been focused on the study of abnormalities in the coagulation cascade, its natural inhibitors and the fibrinolytic system components and both quantitative and qualitative alterations have been demonstrated. Recently the role of platelets and microvascular endothelium has been reviewed, as the possible link between the inflammatory and hemostatic process.

Several studies, in UC and CD patients, reported both quantitative and qualitative alterations of many coagulation enzymes, some of which are also considered acute-phase reactants. This includes elevation of circulating fibrinogen, prothrombin, and factors V, VII, VIII, X, XI and XII [8, 9]. Moreover, many other alterations reflecting a state of hyper-coagulability have been reported in IBD patients, such as elevation of prothrombin factor $1+2$ (side-products of prothrombin cleavage), thrombin-antithrombin complex, fibrinopeptide $A$ and $B$ and decreased factor XIII levels $[10,11]$. These findings are consistent with an activation, which may sometimes be 
subclinical, of coagulation system in IBD. It is still debated if this condition is just a consequence of the inflammatory state or a feature of the intestinal disease per se regardless of disease activity [12].

Endothelial dysfunction has been demonstrated in IBD and markers of its damage such as von Willebrand factor. The knot seems to be an imbalance between nitric oxide, a vasodilator and anti-aggregate agent, and reactive oxygen species, formation in the inflamed endothelium [13].

In IBD patients there are quantitative, morphological and qualitative alterations in platelet characteristic. Thrombocytosis and IBD have been first correlated in 1968 and it is now well established that thrombocytosis is related to disease activity and severity. Thrombocytosis is considered a nonspecific response to inflammation which may occur in chronic inflammatory conditions other than IBD [14].

The circulating concentration of the fibrinolytic system's factors is impaired in UC and CD patients and favors the pro-thrombotic mechanisms. In IBD patients, a reduction in fibrinolysis activators and an increase in inhibitors has been described, inducing a reduced activity of the system $[15,16]$.

Inflammation and venous thromboembolism are linked [17] with inflammation driving venous thrombosis. Inflammation modulates thrombotic responses by upregulating procoagulants, downregulating anticoagulants, and suppressing fibrinolysis. Therefore, autoimmune disorders such as inflammatory bowel disease [18, 19] have been associated with an increased risk of venous thromboembolism. The effects of inflammation on coagulation could all contribute to the identified associations. However, the reduction in risk of pulmonary embolism over time suggests that the thrombotic risk is linked to the inflammatory activity of the autoimmune disorders, which is likely to decrease over time because of treatment.

The therapeutic management of these patients is difficult because of the spontaneous hemorrhages they can exhibit. Massive hemorrhage is seen in $3 \%$ of patients with ulcerative colitis and requires emergency colectomy. Hemorrhage persists in $12 \%$ even after surgery [20] Thrombolytic agents have been used in patients with active ulcerative colitis and massive pulmonary embolism [21] or deep vein thrombosis $[22,23]$ which was the case of our patient. In general, thrombolysis is a life saver to patients who are in cardiogenic shock because of pulmonary embolism, and the counterindications for thrombolytic agents become relative under such circumstances [24]. Even patients with pulmonary embolism, normal blood pressure, but right ventricular dysfunction, benefit from thrombolysis $[25,26]$. Thrombolysis facilitates right ventricular function by breaking up anatomical obstructions, prevents the release of serotonin and other neuro-hormonal factors that exacerbate pulmonary hypertension, and dissolves thrombi in the lower limbs, reducing the probability of recurrence of pulmonary embolism.

The use of anticoagulants requires great care in active $I B D$, because of the risk of causing large hemorrhage [27]. On the other hand, the co-administration of sulphasalazine or azathioprine increases resistance to anticoagulants [28] while the use of glucocorticoids reduces the organism's fibrinolytic action and predisposes to thrombosis. This was the case of our patient. The treatment of patients with active ulcerative colitis and massive pulmonary embolism poses a challenge to the treating physician. In massive pulmonary embolism the significance of contraindications to thrombolysis or other therapeutic interventions is relative and must be weighed against the extreme urgency of the indication.

The glucosaminoglycans are known to have antiinflammatory properties and they facilitate the action of growth factors that are essential for mucosal regeneration and repair [29]. For this reason, heparin has been suggested for the treatment of ulcerative colitis, but its role is controversial. Two main studies addressed this matter; the first [30] showed a benefit from unfractionated heparin, whereas the second not only failed to show a benefit but found an in- 
crease in hemorrhage [31]. The difference between the studies was that in the first the patients were taking mesalazine, as was our patient, while in the second they were not. Low molecular weight heparins do not appear to have a place in the treatment armamentarium for ulcerative colitis. [32] In general, preventive heparin administration is recommended in patients with IBD who are admitted to hospital and remain in a reclining posture for a prolonged period, so that good hydration and rapid mobilization are essential [33]. Our patient was hospitalized before one month before the episode of embolism but did not receive the preventive treatment during the hospitalization.

\section{Conclusion}

Autoimmune disorders in general should be regarded not only as inflammatory disorders, but also as hypercoagulable disorders. Prophylaxis could be warranted in patients admitted with autoimmune disorders or at least for those disorders for which the risk of pulmonary embolism was very high.

\section{Consent}

Written informed consent was obtained from the patient for publication of this Case report and any accompanying images. A copy of the written consent is available for review by the Editor of this journal.

\section{List of abbreviations:}

- IBD: Inflammatory bowel disease.

- UC: Ulcerative colitis

- CD: Crohn's disease.

- VTE: Venous thromboembolism.

\section{Competing interests:}

The author(s) declare that they have no competing interests.

\section{Authors' contributions}

Sahar Mouram conceived the study and participated in the drafting and development work.

Hicham Sabor, Imane Mouram participated in coordination and helped to draft the manuscript. All authors read and approved the final manuscript.

\section{References}

1. White R. The Epidemiology of venous thromboembolism. Circulation 2003; 107 (23 suppl 1): 14-18

2. Goldhaber SZ, Visani L, De Rosa M. Acute pulmonary embolism: clinical outcomes in the International Cooperative Pulmonary Embolism Registry (ICOPER). Lancet 1999; 353: 1386-89.

3. Goldhaber SZ, Elliott CG. Acute pulmonary embolism: part I: epidemiology, pathophysiology, and diagnosis. Circulation 2003; 108: 2726-29.

4. Pogliani EM, Bozzan A, Radaelli F. Thrombolytic therapy for venous thrombosis in patients with gastrointestinal diseases. Pharmatherapeutica. 1982; 3: 268-273.

5. Elikowski W, Malec M, Lawandowska M. Massive pulmonary embolism in a patient with ulcerative colitis and hyperhomocysteinaemia-a case report. Kardio Pol. 2006; 64: 405-409.

6. Novotny DA, Rubin RJ, Slezak FA, et al. Arterial thromboembolic complications of inflammatory bowel disease: report of three cases. Dis Colon Rectum. 1992; 35: 193-196.

7. Solem CA, Loftus EV, Tremaine WJ, et al. Venous thromboembolism in inflammatory bowel disease. Am J Gastroenterol. 2004; 99: 97-101.

8. Chamouard P, Grunebaum L, Wiesel ML, Frey PL, Wittersheim C, Sapin R, et al. Prothrombin fragment $1+2$ and thrombinantithrombin III complex as markers of activation of blood coagulation in inflammatory bowel diseases. Eur J Gastroenterol Hepatol. 1995; 7: 1183-8.

9. Lake AM, Stauffer JQ, Stuart MJ. Hemostatic alterations in inflammatory bowel disease: response to therapy. Am J Dig Dis. 1978; 23: 897-902.

10. Chiarantini E, Valanzano R, Liotta AA, Cellai AP, Fedi S, Ilari I, et al. Hemostatic abnormalities in inflammatory bowel disease. Thromb Res. 1996; 82: 137-46.

11. Smith CJ, Haire WD, Kaufman SS, Mack DR. Determination of prothrombin activation fragments in young patients with inflammatory bowel disease. Am J Gastroenterol. 1996; 91: 1221-5.

12. Van Bodegraven AA. Haemostasis in inflammatory bowel diseases: Clinical relevance. Scand J Gastroenterol. 2003; 239(suppl): 51-62 
13. Magro F, Soares JB, Fernandes D. Venous thrombosis and prothrombotic factors in inflammatory bowel disease. World J Gastroenterol. 2014; 20(17): 4857-72.

14. Grainge J, West J, Card TR. Venous thromboembolism during active disease and remission in inflammatory bowel disease: a cohort study. Lancet. 2010; 375: 657-63.

15. Gris JC, Schved JF, Raffanel C, Dubois A, Aguilar-Martinez P, Arnaud $A$, et al.Impaired fibrinolytic capacity in patients with inflammatory bowel disease. Thromb Haemost. 1990; 63: 472-5.

16. Saibeni S, Bottasso B, Spina L, Bajetta M, Danese S, Gasbarrini A, et al. Assessment of thrombin-activatable fibrinolysis inhibitor (TAFI) plasma levels in inflammatory bowel diseases. Am J Gastroenterol. 2004; 99: 1966-70.

17. $\mathrm{Xu} \mathrm{J,} \mathrm{Lupu} \mathrm{F,} \mathrm{Esmon} \mathrm{CT.} \mathrm{Inflammation,} \mathrm{innate} \mathrm{immunity} \mathrm{and}$ blood coagulation. Hamostaseologie 2010; 30: 5-9.

18. Wang JY, Terdiman JP, Vittinghoff E, Minichiello T, Varma MG. Hospitalized ulcerative colitis patients have an elevated risk of thromboembolic events. World J Gastroenterol 2009; 15: 92735.

19. Grip O, Svensson PJ, Lindgren S. Infl ammatory bowel disease promotes venous thrombosis earlier in life. Scand J Gastroenterol 2000; 35: 619-23.

20. Robert JH, Sachar DB, Aufses AH, Greenstein AJ. Management of severe hemorrhage in ulcerative colitis. Am J Surg. 1990; 159: 550-555.

21. Haagsma CJ, Blom HJ, Van Riel PLCM, et al. Influence of sulphasalazine, methotrexate and the combination of both on plasma homocysteine concentrations in patients with rheumatoid arthritis. Ann Rheum Dis. 1999; 58: 79-84

22. Pogliani EM, Bozzan A, Radaelli F. Thrombolytic therapy for venous thrombosis in patients with gastrointestinal diseases. Pharmatherapeutica. 1982; 3: 268-273.

23. Van Vanwoert JH, Thomson RC, Cangemi JR, Metzger PP, Blackshear JL, Fleming CR. Streptokinase therapy for extensive venous thrombosis in a patient with severe ulcerative colitis. Mayo Clinic Proc. 1990; 65: 1144-1149.

24. Task Force on Pulmonary Embolism of the European Society of Cardiology. Guidelines on diagnosis, management of acute pulmonary embolism. Eur Heart J. 2000; 21: 1301- 1336.

25. Kucher N, Luder CM, Dornhofer T, Windecker S, Meier B, Hess OM. Novel management strategy for patients with suspected pulmonary embolism. Eur Heart J. 2003; 24: 366-376.

26. Kostadima E, Zakynthinos E. Pulmonary embolism: pathophysiology, diagnosis, treatment. Hellenic J Cardiol. 2007; 48: 94-107.

27. Van Vanwoert JH, Thomson RC, Cangemi JR, Metzger PP, Blackshear JL, Fleming CR. Streptokinase therapy for extensive venous thrombosis in a patient with severe ulcerative colitis. Mayo Clinic Proc. 1990; 65: 1144-1149.

28. Teefy AM, Martin JE, Kovacs MJ. Warfarin resistance due to sulfasalazine. Ann Pharmacother. 2001; 35: 506
29. Papa A, Danese A, Gasbarrini A, et al. Review article: potential therapeutic applications and mechanisms of action of heparin in inflammatory bowel disease. Aliment Pharmacol Ther.2000; 14: 1403-1409.

30. Ang YS, Mahmud N, White B, et al. Randomized comparison of unfractionated heparin with corticosteroids in severe active inflammatory bowel disease. Aliment Pharmacol Ther. 2000; 14: 1015-102

31. Panes J, Esteve M, Cabre E, et al. Comparison of heparin and steroids in the treatment of moderate and severe ulcerative colitis. Gastroenterology. 2000; 119: 903-908.

32. Bloom S, Kiilerich S, Lassen MR, et al. Low molecular weight heparin (tinzaparin) vs placebo in the treatment of mild to moderately active ulcerative colitis. Aliment Pharmacol Ther. 2004; 19: 871-878.

33. Carter MJ, Lobo AJ, Travis SP. Guidelines for the management of inflammatory bowel disease in adults. Gut. 2004; 53(Suppl 5): V1-V16.

\section{Comment on this article:}

\section{(f) B in $8+\mathbf{S} P$}

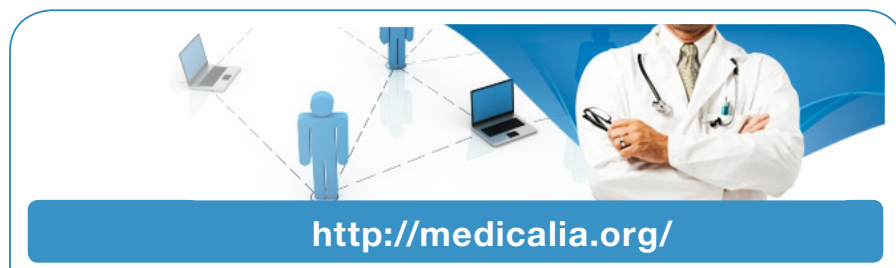

Where Doctors exchange clinical experiences, review their cases and share clinical knowledge. You can also access lots of medical publications for free. Join Now!

\section{Publish with iMedPub}

\section{http://www.imed.pub}

International Archives of Medicine is an open access journal publishing articles encompassing all aspects of medical science and clinical practice. IAM is considered a megajournal with independent sections on all areas of medicine. IAM is a really international journal with authors and board members from all around the world. The journal is widely indexed and classified Q1 in category Medicine. 